\title{
Molecular Orbital Theory of the Electronic Structure of Organic Compounds
}

\section{Dioxodiazacycloalkanes}

\author{
Julio Marañon and Oscar M. Sorarrain ${ }^{1}$ \\ Departamento de Fisica y Química, Facultad de Ciencias Exactas, Universidad Nacional \\ de La Plata, La Plata, Argentina, Miembro Conicet, Argentina \\ Horacio Grinberg, Samuel Lamdan, and Carlos H. Gaozza \\ Departamento de Química Orgánica, Facultad de Farmacia y Bioquímica, \\ Universidad Nacional de Buenos Aires, Buenos Aires, Argentina
}

(Z. Naturforsch. 31 a, $1677-1680$ [1976] ; received September 9, 1976)

The stability of some heterocyclic compounds derived from the condensation of succinic and glutaric anhydrides with aliphatic diamines is compared using calculated values for the total energies. Further, some results derived from the electronic population analysis and the available experimental data are used to give information about the corresponding electronic structure. The CNDO $/ 2$ and INDO methods have been used for the calculations.

\section{Introduction}

In a previous report ${ }^{1}$ we described the generation and spectroscopic properties of the heterocyclic compounds derived from the condensation of succinic and glutaric anhydrides with aliphatic diamines. The principal aim of this paper is to use the semiempirical CNDO/2 and INDO methods for a critical study of the changes in the electronic structure of a dioxodiazacycloalkane caused by the introduction of $\mathrm{CH}_{2}$ groups at either side of the molecule 1 a.

\section{Theoretical Considerations and Used Methods}

Molecular orbital calculations utilizing the $\pi$ electron approximation have been of great use for

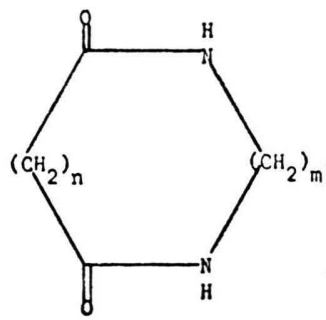

(a)

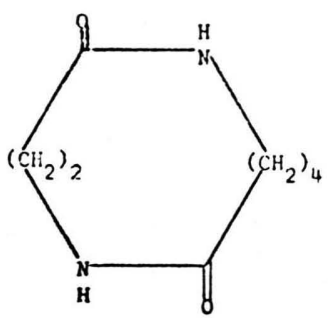

(b)
Fig. 1. a) Dioxodiazacycloalkanes computed; b) 4,10-Dioxo1,5-diazacyclodecane.

Reprint requests to Dr. O. M. Sorarrain, Departemento de Fisica, Calle 115 Y 49 C. C. No 67, La Plata, Argentinien. the understanding of conjugated planar hydrocarbons. The paucity of knowledge concerning $\sigma$-electron distributions and their interactions with the $\pi$ system, would, however, present a serious problem if these calculations were performed on the compounds mentioned above (Fig. 1 a). A selfconsistent method which includes all of the valence shell electrons and explicitly treats the charge separation inherent in the carbonyl group is therefore desired. The complete neglect of differential overlap or $\mathrm{CNDO} / 2$ theory developed by Pople et al. ${ }^{2}$ fulfills these criteria and further requires only the molecular geometry of the molecule in question for input data, thus making it possible to account for conformational differences ${ }^{3}$. The problem of calculating dipole moments from the $\pi$-electron densities and then including standard bond moments for the $\sigma$-framework is obviated since the interaction of the $\sigma$ - and $\pi$-electron system is treated explicitly.

It is difficult to discuss charge distributions within the framework of these molecules according to classical hybridized descriptions of atomic orbitals. This does not represent a theoretical problem since the $\mathrm{CNDO} / 2$ theory was designed specifically ${ }^{2 a}$ so that the electronic structural results of a calculation would be invariant with respect to an orthogonal transformation within the basis set of atomic orbitals. Thus, although the orbitals on the carbon are not couched in the form of trigonal and tetrahedral hybridization, they are linearly combined in a manner which gives the same total charge distribution as would hybridized orbitals. 
Taking into account these considerations, we have undertaken a study of the molecular electronic structure of the dioxodiazacycloalkanes (Fig. $1 \mathrm{a}$ ) by using the semiempirical all valence electrons CNDO/2 and INDO methods.

\section{Results and Discussion}

The total energy of a molecule is a function of both its average electron distribution and nuclear positions. An alteration of the nuclear framework will not only induce changes in the electronic component of the total energy but will also change the inter-nuclear repulsion. In general, then, continuous deformations of the molecular structure produces continuous changes in the total energy. Different configurations have different total energies, the most stable having the lowest value.

Assuming an almost planar conformation, the geometry of the molecules was optimized within the constraints placed upon the molecular parameters we have chose to vary. This was done by interpolation between energies calculated for a number of specific configurations around the minimum energy.

Although the assumption of an almost planar conformation seems to be fairly rigorous, it is justified by the following reasons:

a) The degree of molecular flexibility for 8-11 membered-rings is very similar. Since we are interested in relative values, the planar hypothesis seems to be reasonable.

b) In the case of a single-configuration closed shell wave function (total spin, $S=0$ ), the bond order matrix is invariant to a first approximation for small changes in the molecular geometry ${ }^{4}$.
Derivatives of the energy with respect to the various geometrical parameters can thus be found easily.

c) The total energy of the molecule $1 \mathrm{~b}$ (cyclo peptide) adopting a non planar conformation and minimizing that with respect to the geometrical variables defining its structure gave a minimum of $-3704.70 \mathrm{eV}^{5}$. This value is very close to the corresponding one for the molecule 1 a $(n=3$, $m=4$ ) (Table 1) .

In Table 1 several values are given for the analysed molecules.

Considering the dipole moments, their values are very reasonable excepting perhaps 1 a $(n=2$, $m=2)$. The value (3.769 debyes) seems to be higher than expected due possibly to the Vander Waals repulsion between hydrogen atoms. It can also be observed that for a same number of atoms, the stability as given by the total energy increases with the "molecular symmetry". For instance, 1 a $(n=3, m=4)$ and $(n=3, m=3)$ molecules are more stable than $1 \mathrm{a}(n=2, m=5)$ and $(n=2$, $m=4$ ) molecules, respectively. Finally, the CNDO/2 method gives better values of both the total and bond energies than does the INDO method.

As can be observed in the corresponding electronic density tables the $\mathrm{CNDO} / 2$ method gives the polarizations in good agreement with the electronegativities of the atoms. In particular, the electronic densities of the nitrogen atoms (polarized in the $\mathrm{N}^{-}-\mathrm{H}^{+}$sense) are linearly related with the chemical shifts of the protons linked to them ${ }^{1}$. The equation (found by the minimum squares method) is (see Fig. 2) :

$$
P_{\mathrm{XX}}=-0.033 \delta+5.535 .
$$

Table 1.

\begin{tabular}{llllll}
\hline $\begin{array}{l}\text { Molecule } \\
1 \mathrm{a}\end{array}$ & Method a & $\begin{array}{l}\text { Total } \\
\text { Energy b } \\
-E_{\mathrm{t}}\end{array}$ & $\begin{array}{l}\text { Binding } \\
\text { Energy b } \\
-E_{\mathrm{b}}\end{array}$ & $\begin{array}{l}\text { Ionization } \\
\text { Potential b } \\
I P\end{array}$ & $\begin{array}{l}\text { Dipole } \\
\text { Moment }\end{array}$ \\
\hline $2-2$ & & 2998.6923 & 232.0817 & 12.2368 & 3.769 \\
$2-2$ & INDO & 2884.7382 & 220.6162 & 11.5947 & 4.038 \\
$2-3$ & CNDO/2 & 3156.2381 & 187.1557 & 11.5321 & 1.371 \\
$2-3$ & INDO & 3032.3299 & 171.9940 & 11.0125 & 0.576 \\
$3-2$ & CNDO/2 & 3233.5248 & 264.4421 & 11.9865 & 0.879 \\
$3-2$ & INDO & 3112.5436 & 252.2074 & 11.3063 & 0.952 \\
$2-4$ & CNDO/2 & 3412.5620 & 241.0069 & 11.5784 & 0.348 \\
$2-4$ & INDO & 3281.6088 & 225.0585 & 11.0533 & 0.997 \\
$3-3$ & CNDO/2 & 3466.3933 & 294.8382 & 11.7008 & 1.301 \\
$3-3$ & INDO & 3338.3252 & 281.7746 & 11.0697 & 1.269 \\
$2-5$ & CNDO/2 & 3651.5032 & 277.4760 & 9.7774 & 2.081 \\
$2-5$ & INDO & 3502.3284 & 249.5639 & 9.1653 & 2.263 \\
$3-4$ & CNDO/2 & 3698.3290 & 324.3018 & 11.7226 & 1.520 \\
$3-4$ & INDO & 3563.4695 & 310.7050 & 10.9064 & 1.314 \\
\hline
\end{tabular}

a Calculations were performed on a IBM $360 / 50$;

b eV units;

c Dipole moments are in Debyes. 


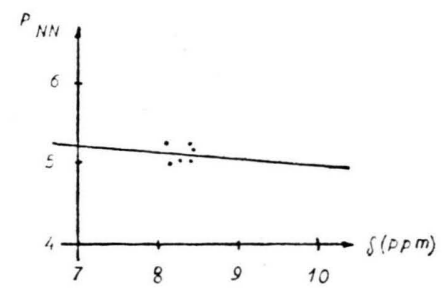

Fig. 2. Electronic population of the nitrogen atoms vs. chemical shifts of the hydrogen atoms linked to them.

Table 2.

(I; $N=2, M=2)$ *

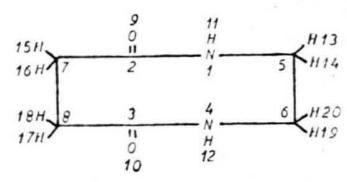

(I; $N=2, M=3) *$

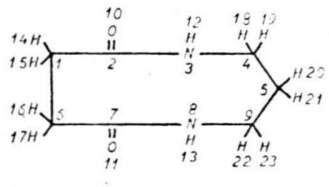

Valence electronic population

\begin{tabular}{rlcl} 
Atom No & $P_{\mathrm{AA}}$ & Atom No & $P_{\mathrm{AA}}$ \\
\hline 1 & 5.2041 & 1 & 4.0555 \\
2 & 3.6327 & 2 & 3.7386 \\
5 & 3.9088 & 3 & 5.3390 \\
7 & 4.0382 & 4 & 3.9433 \\
9 & 6.3740 & 5 & 3.9865 \\
11 & 0.8786 & 10 & 6.3363 \\
13 & 1.0131 & 12 & 6.6613 \\
15 & 0.9674 & 14 & 0.9446 \\
& & 18 & 1.0100 \\
& & 20 & 1.0145 \\
\hline
\end{tabular}

Table 3 .

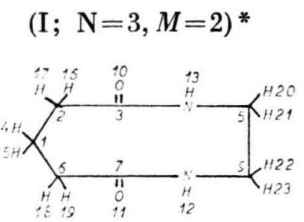

$(\mathrm{I} ; N=3, M=3)$ *

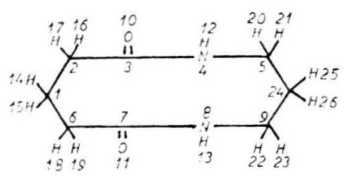

Valence electronic population

\begin{tabular}{rlcl} 
Atom No & $P_{\mathrm{AA}}$ & Atom No & $P_{\mathrm{AA}}$ \\
\hline 1 & 3.9395 & 1 & 3.9364 \\
2 & 4.0221 & 2 & 4.0253 \\
3 & 3.6274 & 3 & 3.6252 \\
4 & 5.2035 & 4 & 5.1916 \\
5 & 3.9155 & 5 & 3.9385 \\
10 & 6.4062 & 10 & 6.4140 \\
13 & 0.8874 & 12 & 0.8780 \\
14 & 1.0143 & 14 & 1.0117 \\
16 & 0.9558 & 16 & 0.9513 \\
20 & 1.0150 & 20 & 1.0145 \\
& & 24 & 3.9590 \\
& & 25 & 1.0136 \\
\hline
\end{tabular}

The results of the electron population analysis (charge densities) are given in the following tables.

We have also analysed the electronic population matrices corresponding to the molecules. In our opinion, there are some results interesting enough to be pointed out:

a) Calculated $\mathrm{C}-\mathrm{H}$ bond orders are close to the values $(0.5)$ corresponding to localized bonds formed from $\mathrm{sp}^{3}$ orbitals.

b) The low value for the long range hydrogenhydrogen bond order agrees with the suggestion

Table 4 .
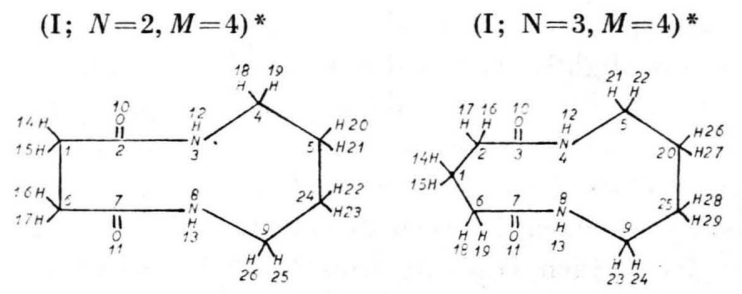

\begin{tabular}{llcl}
\hline & \multicolumn{4}{c}{ Valence electronic population } \\
Atom No & $P_{\mathrm{AA}}$ & Atom No & $P_{\mathrm{AA}}$ \\
\hline 1 & 4.0603 & 1 & 3.9391 \\
2 & 3.7113 & 2 & 4.0259 \\
3 & 5.3369 & 3 & 3.6224 \\
4 & 3.9429 & 4 & 5.1860 \\
5 & 3.9770 & 5 & 3.9465 \\
10 & 6.3368 & 10 & 6.4193 \\
12 & 0.6794 & 12 & 0.8759 \\
14 & 0.9459 & 14 & 1.0082 \\
18 & 1.0119 & 16 & 0.9479 \\
20 & 1.0177 & 20 & 3.9648
\end{tabular}

Table 5 .

\begin{tabular}{|c|c|c|c|}
\hline & & $, M=5)^{*}$ & \\
\hline & & ${ }_{H}^{H}{ }_{24}^{H}$ & \\
\hline & & nic popula & ion \\
\hline Atom No & $P_{\text {AA }}$ & Atom No & $P_{\text {AA }}$ \\
\hline 1 & 4.0652 & 10 & 6.3309 \\
\hline 2 & 3.6894 & 12 & 0.6957 \\
\hline 3 & 5.3410 & 14 & 0.9461 \\
\hline 4 & 3.9024 & 18 & 1.0059 \\
\hline 5 & 4.0840 & 20 & 3.9257 \\
\hline 6 & 4.0655 & 21 & 1.0168 \\
\hline
\end{tabular}


given by Pople et al. ${ }^{6}$ that geminal constants of this sort are small because of cancellation of two effects, the difference between $2 s$ and $2 p$ orbital energies and direct hydrogen-hydrogen bonding.

c) The electronic population of the $2 \mathrm{p}_{y}$ orbital on nitrogen is high (ranging from 1.6994 to 1.8194) corresponding closely to a $\pi$-type lone pair.

d) The large positive element connecting $2 \mathrm{~s}$ orbitals of oxygen (ranging from 0.3804 to 0.4322 ) corresponds to the projection of oxygen lone pair electrons at the back of the molecule and is probably the principal contributor to the calculated dipole moments.

e) The $\pi$-bonding molecular orbital (formed from $2 \mathrm{p}_{z}$ atomic orbitals on carbon and oxygen) is only slightly polarized towards the oxygen.

f) In the $\sigma$-system there is a concentration of oxygen lone pair electrons on the $z$ axis on the side away from the rest of the molecule [shown in the positive element between $2 \mathrm{~s}$ and $2 \mathrm{p}_{z}$ atomic orbitals on the oxygen (ranging from 0.3804 to 0.4322 )]

A further feature of interest in the molecule 1 a $(n=3, m=3)$ and $(n=3, m=2)$ is the substancial

1 H. Grinberg, S. Lamdan, and C. H. Gaozza, J. Heterocyclic Chem. 1975, 783.

2 a) J. A. Pople, D. P. Santry, and G. A. Segal, J. Chem. Phys. 43, S129 [1965] ; b) J. A. Pople and G. A. Segal. ibid. 43, S129 [1965]; c) J. A. Pople and G. A. Segal, ibid. 44, 3289 [1966]. The program written by G. A. bond order (0.1739 and 0.2132 respectively) between $2 \mathrm{p}_{x}$ orbitals on carbon and oxygen. This arises because the oxygen $2 \mathrm{p}_{x}$ orbital (often called the $n$ orbital) is not completely filled, but there is a "back donation" effect in which electrons in orbitals of this symmetry $\left(b_{2}\right)$ are transferred into the antisymmetric $\mathrm{CH}_{2}-\mathrm{C}-\mathrm{NH}$ orbitals forming a partial pseudo- $\pi$ bond or $\pi_{x}$ bond.

Finally, we are willing to supply more information to the readers interested for them.

\section{Acknowledgements}

We are grateful to Alicia Rodriguez Trelles from the Centro de Cómputos de la Faculted de Medicina del Servicio Universitario de Computación for generous amounts of computer time and Alicia Rosales from that Center for her able help with the programs used in this work. J. M. and O. M. S. thank to the O. E. A. and Consejo Nacional de Investigaciones (CONICET). H. G., S. L. and C. H. G. thank to the Universidad de Buenos Aires and CONICET for a research grant in support of this work.

\footnotetext{
Segal was obtained through the Quantum Chemistry Program Exchange at Indiana University.

3 Results to be published.

${ }^{4}$ R. C. Bingham, M. J. S. Dewar, and D. H. Lo, J. Amer. Chem. Soc. 97, 1285 [1975].

5 Unpublished results.

6 J. A. Pople and D. P. Santry, Mol. Phys. 7, 269 [1964].
} 